\title{
Electronic excitations in transition metal dichalcogenide monolayers from an LDA plus GdW approach
}

Drueppel, Matthias; Deilmann, Thorsten; Noky, Jonathan; Marauhn, Philipp; Krueger, Peter; Rohlfing, Michael

Published in:

Physical Review B

Link to article, DOI:

10.1103/PhysRevB.98.155433

Publication date:

2018

Document Version

Publisher's PDF, also known as Version of record

Link back to DTU Orbit

Citation (APA):

Drueppel, M., Deilmann, T., Noky, J., Marauhn, P., Krueger, P., \& Rohlfing, M. (2018). Electronic excitations in transition metal dichalcogenide monolayers from an LDA plus GdW approach. Physical Review $B, 98(15)$, [155433 ]. https://doi.org/10.1103/PhysRevB.98.155433

\section{General rights}

Copyright and moral rights for the publications made accessible in the public portal are retained by the authors and/or other copyright owners and it is a condition of accessing publications that users recognise and abide by the legal requirements associated with these rights.

- Users may download and print one copy of any publication from the public portal for the purpose of private study or research.

- You may not further distribute the material or use it for any profit-making activity or commercial gain

- You may freely distribute the URL identifying the publication in the public portal 


\title{
Electronic excitations in transition metal dichalcogenide monolayers from an LDA $+G d W$ approach
}

\author{
Matthias Drüppel, ${ }^{1}$ Thorsten Deilmann, ${ }^{2}$ Jonathan Noky, ${ }^{3}$ Philipp Marauhn, ${ }^{1}$ Peter Krüger, ${ }^{1}$ and Michael Rohlfing ${ }^{1, *}$ \\ ${ }^{1}$ Institut für Festkörpertheorie, Westfälische Wilhelms-Universität Münster, 48149 Münster, Germany \\ ${ }^{2}$ Center for Atomic-Scale Materials Design (CAMD), Department of Physics, Technical University of Denmark, \\ DK-2800 Kongens Lyngby, Denmark \\ ${ }^{3}$ Max-Planck-Institute for Chemical Physics of Solids, 01187 Dresden, Germany
}

(Received 4 July 2018; published 23 October 2018)

\begin{abstract}
Monolayers of transition metal dichalcogenides (TMDCs) have unique optoelectronic properties. Density functional theory allows only for a limited description of the electronic excitation energies in these systems, while a more advanced treatment within many-body perturbation theory employing the $G W / \mathrm{BSE}$ approximation is often rather time consuming. Here, we show that the recently developed LDA $+G d W$ approach provides an efficient and at the same time reliable description of one-particle energies, as well as optical properties including bound excitons in TMDCs. For five exemplary materials $\left(\mathrm{MoSe}_{2}, \mathrm{MoS}_{2}, \mathrm{WSe}_{2}, \mathrm{WS}_{2}\right.$, and $\left.\mathrm{ReSe}_{2}\right)$, we discuss the numerical convergence, in particular with respect to k-point sampling, and show that the $G d W / \mathrm{BSE}$ approximation gives results similar to common $G W / \mathrm{BSE}$ calculations. Such efficient approaches are essential to treat larger multilayer systems or defects.
\end{abstract}

DOI: 10.1103/PhysRevB.98.155433

\section{INTRODUCTION}

Transition metal dichalcogenides (TMDCs) have recently attracted tremendous attention due to their remarkable electronic and optical properties. The bulk crystal of a TMDC is formed by individual layers that are weakly held together by van der Waals interaction in the perpendicular direction, but also few and even monolayers can be exfoliated. One of the most widely studied TMDCs is $\mathrm{MoS}_{2}$, a material with an indirect band gap in the bulk, which becomes direct in the monolayer limit $[1,2]$. The two-dimensional nature of the monolayer leads to a strong confinement and a drastically reduced screening compared to bulk semiconductors, which results in strongly bound excitons [3-6]. These can be selectively excited by circularly polarized light in one of the two $K$ valleys of the Brillouin zone $[7,8]$.

Theoretical insights into the electronic and optical properties of TMDCs can be obtained by many-body perturbation theory (MBPT), i.e., the $G W$ method followed by the solution of the Bethe-Salpeter equation (BSE), which is a state-of-theart method to reliably describe excited electronic states in a parameter-free way $[9,10]$.

One disadvantage of a $G W$ calculation is its high numerical cost, especially in TMDCs [11,12]. These numerical restrictions put systems with large unit cells out of reach and already make it costly to obtain converged results for the regular unit cell (three atoms). The high numerical demands of a $G W$ calculation in TMDC monolayers are reflected in the significant spread of the results within the published works on their optoelectronic properties [3-5,11-16]. Two examples are the exciton binding energy with reported values ranging from 0.5 [5] to $1.1 \mathrm{eV} \mathrm{[4]} \mathrm{and} \mathrm{the} \mathrm{optical} \mathrm{gap} \mathrm{(i.e.,} \mathrm{the} \mathrm{energy}$

*michael.rohlfing@uni-muenster.de of the $A$ exciton) with reported values between $1.78[3]$ and $2.22 \mathrm{eV}$ [13]. In addition, there have been electronic structures of monolayer $\mathrm{MoS}_{2}$ published showing both an indirect band gap $[11,13]$ as well as a direct band gap $[3,4,16-18]$ while experimental results strongly suggest a direct gap $[1,19,20]$.

To drastically decrease the numerical demands of a $G W / B S E$ calculation, Rohlfing proposed a way of conducting $a b$ initio MBPT with an additional approximation in the selfenergy: the LDA $+G d W$ approach, which can also be combined with the BSE to evaluate optical excitations [21]. In this work we apply the LDA $+G d W / \mathrm{BSE}$ approach to five TMDC monolayers: $\mathrm{MoSe}_{2}, \mathrm{MoS}_{2}, \mathrm{WSe}_{2}, \mathrm{WS}_{2}$, and $\mathrm{ReSe}_{2}$. We find results in good agreement with theoretical outcomes based on the $G W / \mathrm{BSE}$ method as well as experimental findings.

Here, we discuss in particular the convergence behavior of the band structures and the optical excitation spectra. Some of these issues, like the size of the auxiliary basis set, are specific for the perturbative treatment of the $G W$ self-energy operator within our LDA $+G d W$ method of MBPT. More importantly, we demonstrate that by exactly matching the reciprocal-space sampling techniques for the quasiparticle energies and optical excitations to each other, rapid k-point convergence is achieved. This behavior is a generic feature of MBPT, representing the direct correspondence between the self-energy operator and the electron-hole interaction kernel, and is therefore of universal significance for all methods linking single-particle and two-particle excitations to each other. Also, these features are not restricted to two-dimensional semiconductors, but hold for all periodic condensed matter.

It is important to note that our LDA $+G d W$ approximation may not reach the accuracy of $\sim 0.1 \mathrm{eV}$ for absolute energies commonly attributed to the $G W$ or $G W / \mathrm{BSE}$ method. Nevertheless, it allows for precise calculations of relative energies as resulting from differences between related systems, e.g., system-specific states of nanostructures (like surface states as 
compared to bulk states), and modifications due to changes in the nonlocal dielectric polarizability. Such issues can be fully discussed within $\mathrm{LDA}+G d W$, which offers higher numerical efficiency than the conventional GWA.

The paper is organized as follows: In Sec. II we recapitulate the LDA $+G d W$ method, the employed dielectric model functions, and further details of the numerical implementation. Afterwards, we discuss the numerical execution and its convergence in Sec. III. Finally, we present the results for five TMDC monolayers in Sec. IV.

\section{THEORETICAL METHODS}

In the last decades, a wide range of first-principle methods have been established. For the most successful and widely used density functional theory (DFT), some drawbacks have been identified (like the band-gap problem). On the other hand, many-body perturbation theory in the $G W$ approximation [22] overcomes many of these problems at the price of a significantly enhanced numerical effort.

Instead of relying on an exchange-correlation potential $V_{\mathrm{xc}}$ in DFT the $G W$ method introduces the self-energy $\Sigma=i G W$ (see e.g. [23,24]). The Green function $G$ can be reliably represented using DFT results and the screened Coulomb interaction $W$ is typically calculated using the random-phase approximation (RPA). The RPA, however, is often the computationally most expensive part of the approach and so a reliable approximative calculation of $W$ is greatly appreciated. Furthermore, a frequency integration contained in the evaluation of $\Sigma$ has to be treated. This can be carried out using plasmon-pole expansions, full-frequency integration methods, or resolvent approaches [25]. In this work we focus on a simple plasmon-pole model $[23,24]$ which only requires the evaluation of the dielectric function at two frequency points $\omega=0$ and $\omega=i$ Ryd.

\section{A. LDA $+G d W$ approximation: An efficient approach to the quasiparticle corrections}

In our quasiparticle approach we use a DFT calculation within the local density approximation (LDA) as starting point. This is followed by the $G W$ approach within the LDA $+G d W$ approximation, which is described briefly below. A complete introduction is given in Ref. [21].

In conventional GWA (on top of DFT-LDA), the quasiparticle energies $E_{n \mathbf{k}}^{\mathrm{QP}}$ are obtained via

$$
\begin{aligned}
E_{n \mathbf{k}}^{\mathrm{QP}} & =E_{n \mathbf{k}}^{\mathrm{LDA}}+\left\langle n \mathbf{k}\left|\Sigma\left(E_{n \mathbf{k}}^{\mathrm{QP}}\right)-V_{\mathrm{xc}}\right| n \mathbf{k}\right\rangle \\
& =E_{n \mathbf{k}}^{\mathrm{LDA}}+\left\langle n \mathbf{k}\left|\Sigma\left(E_{n \mathbf{k}}^{\mathrm{QP}}\right)\right| n \mathbf{k}\right\rangle-\left\langle n \mathbf{k}\left|V_{\mathrm{xc}}\right| n \mathbf{k}\right\rangle .
\end{aligned}
$$

The exchange correlation (xc) effects are described by the xc potential $V_{\mathrm{xc}}$, which is then replaced by the self-energy $\Sigma=i G W$. Therefore, it becomes necessary to subtract $V_{\mathrm{xc}}$ from the DFT results. Since there is no closed expression for $\Sigma-V_{\mathrm{xc}}$, both terms have to be evaluated separately and the difference is taken afterwards. This means that the small size of the difference (usually $<1 \mathrm{eV}$ ) between $\Sigma$ and $V_{\mathrm{xc}}$ is not exploited. Both quantities are often in the order of $10 \mathrm{eV}$ or more and have to be converged independently. To obtain quasiparticle energies that are converged better than
$0.1 \mathrm{eV}$, the numeric precision in $\left\langle\Sigma\left(E_{n \mathbf{k}}^{\mathrm{QP}}\right)\right\rangle$ alone needs to be better than $1 \%$, which is difficult to achieve and is thus partly responsible for the high numerical demands in the $G W$ approximation.

The key to the LDA $+G d W$ approach is the approximation of the difference $\Sigma-V_{\text {xc }}$ by a closed expression. This is made possible through the observation that in many systems the LDA $V_{\mathrm{xc}}$ potential acts like a self-energy with a metalliclike screening [26-28]. This implies that $V_{\mathrm{xc}} \approx i G W_{\text {metal }}$ is a good approximation, where $W_{\text {metal }}$ is the metallically screened Coulomb interaction. Such behavior has been observed in $G W$ calculations with artificially metallic screening, and it is also consistent with the common observation that quasiparticle corrections for metals are very small [26-28]. Note that this screening is hypothetical, but can be constructed even for a material which is a semiconductor in reality (see below). Now, $V_{\mathrm{xc}} \approx i G W_{\text {metal }}$ turns $\hat{\mathcal{H}}^{G W}=\hat{\mathcal{H}}^{\mathrm{LDA}}+i G W-V_{\mathrm{xc}}$ into

$$
\begin{aligned}
\hat{\mathcal{H}}^{G d W} & :=\hat{\mathcal{H}}^{\mathrm{LDA}}+i G W-i G W_{\text {metal }} \\
& =\hat{\mathcal{H}}^{\mathrm{LDA}}+\overbrace{i G \underbrace{\Delta \Sigma\left(W-W_{\text {metal }}\right)}_{d W}}^{\Delta \Sigma},
\end{aligned}
$$

where $W$ is the real (e.g., semiconducting) screening of the system. Note that in Eq. (2) we are not separately evaluating $i G W$ and $V_{\mathrm{xc}}$ but rather $i G d W$, which contains the difference in screening $d W$. This approach is very appealing from a numerical point of view since $\langle i G d W\rangle$ is in the order of the quasiparticle corrections, i.e., $\sim 1 \mathrm{eV}$. Converging this quantity to $0.1 \mathrm{eV}$ demands a numerical precision of $10 \%$ only.

The concept of deriving $\Delta \Sigma$ from the difference between semiconducting and metallic screening constitutes a big advantage in the case of TMDC materials. In conventional $G W$ calculations for $d$-electron systems, the $s$ and $p$ semicore states of the same shell must commonly be included explicitly as valence states in the calculation, significantly slowing down the numerical performance $[11,15]$. For example, molybdenum requires a valence-state configuration of $4 s^{2} 4 p^{6} 4 d^{5} 5 s^{1}$ with 14 electrons. This is due to the much different treatment of exchange interaction in $V_{x c}$ and $\Sigma^{G W}$. Within our LDA $+G d W$ approach, on the other hand, $\Delta \Sigma$ as defined in Eq. (2) only consists of a medium- and longranged modification of the dielectric screening, which is not affected by the semicore states. Consequently, we can employ soft pseudopotentials for a configuration $4 d^{5} 5 s^{1}$, i.e., with six electrons only. This significantly accelerates all calculations.

Note that the requirement $V_{\mathrm{xc}} \approx i G W_{\text {metal }}$ should be carefully checked for every system. This comparison can be conducted for small unit cells before increasing the unitcell size in $\mathrm{LDA}+G d W$ to be able to handle more complex systems. Applicability of the LDA $+G d W$ approximation with excellent results was so far shown on a variety of systems with different dimensionality: bulk silicon and its (111) surface, bulk argon, an argon monolayer on aluminum (001) [21], organic molecules on surfaces [29], TMDC bulk, and monolayers [30-34]. 


\section{B. Model dielectric function}

Within $\mathrm{LDA}+G d W$, we exploit that the small size of $i G W-V_{\mathrm{xc}} \approx i G d W$ lowers the demands on the numerical precision. This allows the inclusion of additional approximations like a model dielectric function $\varepsilon\left(\mathbf{r}, \mathbf{r}^{\prime}, \omega\right)$ for the screening. In full $G W$ calculations this would be more problematic. Within the $G W$ approxiamtion (GWA), $\varepsilon$ is usually calculated through the random phase approximation, which often constitutes a bottleneck of the calculation; thus, the model function drastically reduces computation time.

The use of a model function that can address both semiconducting and metallic screening is mandatory since we need to evaluate the difference in screening, ( $\left.W-W_{\text {metal }}\right)$. This is only useful if both are described through the same model-function structure. One requirement of the used model function is that it can describe spatially inhomogeneous screening. Rohlfing [21] proposed a model which constructs the screening from a summation of charge-density responses $\chi^{j}$ of each atom $j$ in the unit cell

$$
\varepsilon_{\mathbf{G}, \mathbf{G}^{\prime}}(\mathbf{q}, \omega)=\delta_{\mathbf{G}, \mathbf{G}^{\prime}}+\frac{4 \pi e^{2}}{\left|\mathbf{q}+\mathbf{G} \| \mathbf{q}+\mathbf{G}^{\prime}\right|} \sum_{j=1}^{N} \frac{V_{j}}{V} \chi_{\mathbf{G}, \mathbf{G}^{\prime}}^{j}(\mathbf{q}, \omega)
$$

where $\chi^{j}$ gets weighted by the volume $V_{j}$ of the respective atom, divided by the volume of the unit cell $V$ (in the bulk). The expression above uses an expansion in a plane-wave basis $\mathbf{G}$. The prefactor $4 \pi e^{2} /\left(\left|\mathbf{q}+\mathbf{G} \| \mathbf{q}+\mathbf{G}^{\prime}\right|\right)$ stems from the convolution with the Coulomb interaction.

The dielectric model function has been discussed in detail in Ref. [21]. The charge-density response resulting from atom $j$ at position $\boldsymbol{\tau}_{j}$ is assumed as

$$
\begin{aligned}
\chi_{\mathbf{G}, \mathbf{G}^{\prime}}^{j}(\mathbf{q}, \omega)= & \sum_{k=1}^{3} \sqrt{f^{(j, k)}(|\mathbf{q}+\mathbf{G}|, \omega) f^{(j, k)}\left(\left|\mathbf{q}+\mathbf{G}^{\prime}\right|, \omega\right)} \\
& \times\left[(\mathbf{q}+\mathbf{G}) \mathbf{n}_{j}^{k}\right] \cdot\left[\mathbf{n}_{j}^{k}\left(\mathbf{q}+\mathbf{G}^{\prime}\right)\right] \\
& \times e^{-\gamma_{j}\left(\mathbf{G}^{\prime}-\mathbf{G}\right)^{2}} e^{i\left(\mathbf{G}^{\prime}-\mathbf{G}\right) \boldsymbol{\tau}_{j}}
\end{aligned}
$$

in the nonmetallic case, while the metallic response function is given isotropically by

$$
\begin{aligned}
\chi_{\mathbf{G}, \mathbf{G}^{\prime}}^{j}(\mathbf{q}, \omega)= & \sqrt{f^{(j)}(|\mathbf{q}+\mathbf{G}|, \omega) f^{(j)}\left(\left|\mathbf{q}+\mathbf{G}^{\prime}\right|, \omega\right)} \\
& \times|\mathbf{q}+\mathbf{G}| \cdot\left|\mathbf{q}+\mathbf{G}^{\prime}\right| \\
& \times e^{-\gamma_{j}\left(\mathbf{G}^{\prime}-\mathbf{G}\right)^{2}} e^{i\left(\mathbf{G}^{\prime}-\mathbf{G}\right) \boldsymbol{\tau}_{j}} .
\end{aligned}
$$

Here, $\mathbf{n}_{j}^{k}$ denotes the three principal axes of the $3 \times 3$ dielectric tensor for atom $j$ with associated eigenvalues $\varepsilon^{(j, k)}$. For isotropic systems the response is identical in all directions $k$ and this index is skipped. These expressions rely on the charge-density response of a homogeneous system which was proposed by Bechstedt et al. [35] and is given by

$$
f^{(j, k)}(Q, \omega)=\left[\frac{1}{\varepsilon_{\infty}-1}+\frac{Q^{2}}{q_{\mathrm{TF}}^{2}}+\frac{Q^{4}}{\omega_{\mathrm{p}}^{2}}\right]^{-1},
$$

TABLE I. Atom-resolved dielectric-screening parameters $\varepsilon_{\infty}^{(j, \|)}(\omega=0)$ (for in-plane response) and $\varepsilon_{\infty}^{(j, \perp)}(\omega=0)$ (for response perpendicular to the plane), as well as the Thomas-Fermi wave number $q_{\mathrm{TF}}^{(j)}$ and the plasmon frequency $\omega_{\mathrm{p}}^{(j)}$ (in a.u.). The $\varepsilon$ values have been calculated within the RPA and are used as parameters for the dielectric screening in LDA $+G d W$ [Eqs. (4)-(6)]. Note that $\varepsilon_{\infty}$ commonly refers to frequency much below electronic transitions but much higher than phonons, i.e., $\varepsilon_{\infty}$ refers to a rigid lattice.

\begin{tabular}{lccccc}
\hline \hline & & $\varepsilon_{\infty}^{(j, \|)}(\omega=0)$ & $\varepsilon_{\infty}^{(j, \perp)}(\omega=0)$ & $q_{\mathrm{TF}}^{(j)}$ & $\omega_{\mathrm{p}}^{(j)}$ \\
\hline $\mathrm{MoS}_{2}$ & $\mathrm{Mo}$ & 10.1 & 5.2 & 1.20 & 1.58 \\
& $\mathrm{~S}$ & 11.8 & 7.6 & 1.20 & 1.58 \\
$\mathrm{MoSe}_{2}$ & $\mathrm{Mo}$ & 10.8 & 5.0 & 1.18 & 1.49 \\
& $\mathrm{Se}$ & 10.6 & 6.5 & 1.18 & 1.49 \\
$\mathrm{WS}_{2}$ & $W$ & 10.5 & 5.3 & 1.20 & 1.59 \\
& $\mathrm{~S}$ & 10.9 & 7.5 & 1.20 & 1.59 \\
$\mathrm{WSe}_{2}$ & $W$ & 11.3 & 5.4 & 1.18 & 1.49 \\
& $\mathrm{Se}$ & 9.6 & 6.5 & 1.18 & 1.49 \\
$\mathrm{ReSe}_{2}$ & $\mathrm{Re}$ & $7.6-12.0$ & $4.6-4.8$ & 1.20 & 1.59 \\
& $\mathrm{Se}$ & $8.9-12.1$ & $5.6-6.7$ & 1.17 & 1.47 \\
& & \multicolumn{4}{c}{}
\end{tabular}

where the parameters are given per atom $j$, direction $k$, and frequency $\omega$ :

$\varepsilon_{\infty}=\varepsilon_{\infty}^{(j, k)}(\omega), \quad q_{\mathrm{TF}}^{2}=\left[q_{\mathrm{TF}}^{(j)}\left(\bar{\rho}^{(j)}\right)\right]^{2}, \quad \omega_{\mathrm{p}}^{2}=\left[\omega_{\mathrm{p}}^{(j)}\left(\bar{\rho}^{(j)}\right)\right]^{2}$.

In here, the plasma frequency $\omega_{\mathrm{p}}=\sqrt{16 \pi \bar{\rho}}$ and the ThomasFermi wave number $q_{\mathrm{TF}}=2 \sqrt[6]{3 \bar{\rho} / \pi}$ (in atomic units) both depend on the average valence-electron density $\bar{\rho}$ of the respective atom.

Table I lists characteristic parameters for the systems discussed in this paper. The dielectric screening parameters are obtained from RPA calculations of $\varepsilon_{\mathbf{0}, \mathbf{0}}(\mathbf{q} \rightarrow 0, \omega)$, divided into contributions of each atom [21]. Note that due to the anisotropy of the material, different parameters are found for in-plane and out-of-plane dielectric properties. In combination, they would also lead to the different dielectric constants (for ordinary and extraordinary field geometries) of bulk TMDC materials. The larger in-plane numbers reflect the higher flexibility of electronic displacement within the plane, as compared to the higher rigidity in the perpendicular direction. In the case of $\mathrm{ReSe}_{2}$, which has a distorted structure formed from diamond-shaped elements, the nonequivalence of the various atoms causes variation in the dielectric screening parameters. In all cases, the dielectric screening parameters show a plasmon-pole-like frequency dependence (not shown in Table I) as commonly found in semiconductors in general. In the case of metallic screening (necessary for determining the difference in screening as the heart of the LDA $+G d W$ approach), we simply set $\epsilon_{\infty}$ to $\infty$ in Eq. (5). Thus, only the volume $V_{j}$, the average valence-electron density $\bar{\rho}_{j}$, and the spatial extent $\gamma_{j}$ of each atom need to be chosen manually. However, we want to stress that the final result, i.e., the LDA $+G d W$ band structure, often shows little dependence on these parameters since they appear both in $W$ as well as $W_{\text {metal }}$ and only the difference is evaluated. 


\section{Numerical efficiency of the LDA $+G d W$ approach}

As mentioned above, the LDA $+G d W$ approximation offers a drastic reduction of the numerical demands compared to a full $G W$ calculation [21]: (i) It allows (and requires) the usage of a model dielectric function, eliminating the need for timeconsuming calculations of the full $\varepsilon$ in the random phase approximation. Only some matrix elements $\varepsilon_{\mathbf{0}, \mathbf{0}}(\mathbf{q} \rightarrow 0, \omega)$ have to be evaluated within the RPA to determine the parameters entering the model. (ii) The smallness of $i G\left(W-W_{\text {metal }}\right)$ is exploited, which dramatically facilitates the evaluation within the desired precision, e.g., with respect to the basis size and the applied k-point grids. The reduced demands on the basis result from the fact that while $W$ and $W_{\text {metal }}$ are both highly structured in real space, their difference $\left(W-W_{\text {metal }}\right)$ is much smoother and can thus be well described by a comparably small basis set. (iii) The number of empty bands required for a converged calculation of the self-energy operator $\Sigma$ is often reduced by orders of magnitude, which is also a result of the smooth spatial structure of ( $\left.W-W_{\text {metal }}\right)$.

Finally, we want to underline that the $\mathrm{LDA}+G d W$ approximation, just as the GWA, goes far beyond DFT through the quasiparticle description, resulting in conceptually meaningful band structure gaps and dispersions. Most importantly, inhomogeneous screening is treated with atom-by-atom resolution, including long-range polarization effects that are captured by the nonlocal screened Coulomb interaction $W\left(\mathbf{r}, \mathbf{r}^{\prime}\right)$, a point completely absent in DFT.

\section{Reciprocal-space sampling}

In this section we discuss the various k-point samplings that occur in any $G W / \mathrm{BSE}$ approach for a periodic system. We underline that the discussion holds for 1D, 2D, and 3D systems although we focus on 2D materials in this work.

(1) An exciton state is in principle expanded as

$$
|S\rangle=\int d^{3} k \sum_{v, c} A_{v, c}^{(S)}(\mathbf{k})|(v \mathbf{k}) \rightarrow(c, \mathbf{k}+\mathbf{Q})\rangle,
$$

i.e., as a coherent superposition of independent interband transitions, with $v / c$ denoting occupied and empty bands, $\mathbf{Q}$ being the total momentum of the exciton (often close to zero), and the k-space integration covering the first Brillouin zone. In numerical practice, this integration is commonly replaced by a summation over a finite number of $\mathbf{k}$ points:

$$
|S\rangle=\sum_{\mathbf{k}_{i}} \sum_{v, c} A_{v, c}^{(S)}\left(\mathbf{k}_{i}\right)\left|\left(v \mathbf{k}_{i}\right) \rightarrow\left(c, \mathbf{k}_{i}+\mathbf{Q}\right)\right\rangle .
$$

Each $\mathbf{k}_{i}$ represents a volume $V_{i}$ in reciprocal space (usually all $V_{i}$ are of equal size and shape). We note that the expansion coefficient $A\left(\mathbf{k}_{i}\right)$ represents the average of the original ones, $A\left(\mathbf{k}_{i}\right)=1 / V_{i} \int_{V_{i}} A(\mathbf{k}) d^{3} k$. A finite sampling makes only sense if $A(\mathbf{k})$ does not vary too much within $V_{i}$. This set of $\mathbf{k}$ points defines all requirements to be discussed below.

A simple example would be a Monkhorst-Pack grid, as indicated in Fig. 1(a) by the solid dots. The grid may include any desired shift relative to the origin. If an exciton carries a finite total momentum $\mathbf{Q}$, the hole and electron states are shifted relative to each other by $\mathbf{Q}$ in reciprocal space. In that case, the respective band structure energies are requested
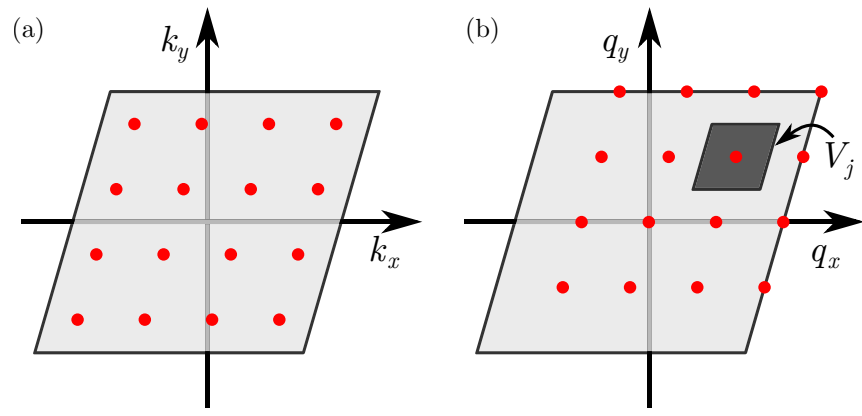

FIG. 1. General scheme of reciprocal-space sampling of a periodic system. (a) Finite grid of $\mathbf{k}_{i}$ points that are used to represent excitonic states. The figure shows a regular grid (e.g., a MonkhorstPack grid), but grids with variable spacing are also possible, in principle. (b) Finite grid of $\mathbf{q}_{j}$ points representing the differences between the $k$ points of (a). Each $\mathbf{q}_{j}$ point represents a little reciprocal-space volume $\left(V_{j}\right)$ necessary for averaging $W(\mathbf{q})$ (see text). In case of a $N_{1} \times N_{2} \times N_{3}$ Monkhorst-Pack grid, $V_{j}$ is given by the Brillouin zone divided by $N_{j}$ in reciprocal-space direction $j=1,2,3$.

on the same grid for the hole states and on the same grid (but shifted by $\mathbf{Q}$ ) for the electron states. In this study, we concentrate on excitons with $\mathbf{Q} \approx 0$ which may be optically active.

(2) The BSE for an exciton given by Eq. (7) and its excitation energy $\Omega$ is given (omitting band indices for brevity sake, and disregarding electron-hole exchange at the moment) by

$$
\Delta E(\mathbf{k}) A(\mathbf{k})-\int W\left(\mathbf{k}-\mathbf{k}^{\prime}\right) A\left(\mathbf{k}^{\prime}\right) d^{3} \mathbf{k}^{\prime}=\Omega A(\mathbf{k})
$$

with $\Delta E(\mathbf{k})$ denoting band-energy differences and $W(\mathbf{q})$ denoting the screened Coulomb interaction. Note that $W(\mathbf{k}-$ $\mathbf{k}^{\prime}$ ) contains further spatial information, either in terms of a Fourier transform $\left[W_{\mathbf{G}, \mathbf{G}^{\prime}}(\mathbf{q})\right.$, cf. Eq. (3)] or, after multiplication with single-particle wave functions, in terms of band indices [cf. Eq. (12); see, e.g., Ref. [10]]. The indices are omitted here for brevity sake. When representing the exciton as a sum over a finite set of $\mathbf{k}$ points [Eq. (8)], the BSE turns into

$$
\Delta E\left(\mathbf{k}_{i}\right) A\left(\mathbf{k}_{i}\right)-\sum_{j} \tilde{W}\left(\mathbf{k}_{i}-\mathbf{k}_{j}\right) A\left(\mathbf{k}_{j}\right)=\Omega A\left(\mathbf{k}_{i}\right) .
$$

In here,

$$
\tilde{W}\left(\mathbf{k}_{i}-\mathbf{k}_{j}\right):=1 / V_{j} \int_{V_{j}} W\left(\mathbf{k}_{i}-\mathbf{k}^{\prime}\right) d^{3} \mathbf{k}^{\prime}
$$

is the integral of $W(\mathbf{q})$ over a (little) volume $V_{j}$ around $\left(\mathbf{k}_{i}-\mathbf{k}_{j}\right)$, fully considering the shape and size of $V_{j}$ [see Fig. 1(b)]. This integration is performed using the reciprocalspace representation $W_{\mathbf{G}, \mathbf{G}^{\prime}}(\mathbf{q})$. For each $\mathbf{G}, \mathbf{G}^{\prime}$ the Coulomb interaction $4 \pi e^{2} /\left(\left|\mathbf{q}+\mathbf{G} \| \mathbf{q}+\mathbf{G}^{\prime}\right|\right)$ is integrated on a very fine three-dimensional reciprocal-space grid of the integration volume $V_{j}$ given by Eq. (11), with $\mathbf{q}=\mathbf{k}_{i}-\mathbf{k}^{\prime}$. Thereafter, convolution with the inverse dielectric matrix at $\mathbf{q}=\mathbf{k}_{i}-\mathbf{k}_{j}$ yields $\tilde{W}$. So far, this procedure disregards the $\mathbf{q}$ dependence of $\varepsilon$ within $V_{j}$, in particular for $\mathbf{G}=\mathbf{G}^{\prime}=0$, and especially for $\mathbf{k}_{i}=\mathbf{k}_{j}$. This is now incorporated (for $\mathbf{G}=\mathbf{G}^{\prime}=0$ ) by 
approximating $\varepsilon(\mathbf{q}) \approx \sum_{i=1}^{3} \bar{\varepsilon}_{i} q_{i}^{2} / q^{2}$ where $\bar{\varepsilon}_{i}$ are the principal values of the macroscopic dielectric tensor at $\mathbf{q}=0$ and $q_{i}$ the respective components of $\mathbf{q}$. With this assumption, the integrand of Eq. (11) reads as $4 \pi e^{2} /\left(q^{2} \varepsilon(\mathbf{q})\right)$ and is again integrated on the very fine three-dimensional reciprocal-space grid mentioned above. Numerical convergence may require millions of grid points, in particular for $\mathbf{k}_{i}=\mathbf{k}_{j}$ due to the Coulomb divergence for $\mathbf{q} \rightarrow 0$, but the total cost of these operations is still moderate compared to that of the rest of our approach.

For large reciprocal-space distance, $\tilde{W}\left(\mathbf{k}_{i}-\mathbf{k}_{j}\right) \approx$ $W\left(\mathbf{k}_{i}-\mathbf{k}_{j}\right)$. For small reciprocal-space distance, however, there are drastic differences between the two expressions. For instance, if the volume $V$ were a sphere of radius $K$ (which, however, does not occur for periodic systems) and $W(\mathbf{q})=\left(4 \pi e^{2}\right) /\left(\epsilon_{\infty} q^{2}\right)$, we would obtain $\tilde{W}(\mathbf{q}=0)=$ $\left(12 \pi e^{2}\right) /\left(\epsilon_{\infty} K^{2}\right)$ while the "true" $W(\mathbf{q} \rightarrow 0)$ diverges. This example illustrates a crucial issue: Forcing the BSE into a finite $\mathbf{k}$-point grid is equivalent to modifying the screened Coulomb interaction, in particular its long-ranged behavior (i.e., at small q). Note that anisotropy in $\varepsilon(\mathbf{q})$ fully enters $\tilde{W}(\mathbf{q})$.

(3) For consistency with the k-point set of the BSE, the finite q-point set on which $\tilde{W}$ is required is defined as all the differences among the $k_{i}$ [see Fig. 1(b)]. The grid thus has the same structure and grid density, but is not shifted with respect to the origin: it contains $q=0$. The grid may also contain points on the boundary of the Brillouin zone (e.g., for an even number of grid points in one direction of a Monkhorst-Pack grid). Due to the periodicity of $\tilde{W}\left(\mathbf{q}_{i}\right)$, points on opposite boundaries of the Brillouin zone are shifted relative to each other by a reciprocal lattice vector $\mathbf{G}$, contain the same physical information, and only one out of a pair or group is taken into account [as illustrated in Fig. 1(b)].

(4) A very crucial feature of MBPT is the one-to-one correspondence between the $G W$ self-energy operator $\Sigma^{G W}$ and the direct part of the corresponding electron-hole interaction kernel derived from $\Sigma^{G W}$ :

$$
\begin{aligned}
\Sigma(1,2) & =i G(1,2) W\left(1^{+}, 2\right) \\
\Longrightarrow K^{d}(13,24) & =\partial \Sigma(1,2) / \partial G(4,3) \\
& =i W\left(1^{+}, 2\right) \delta(1,4) \delta(2,3),
\end{aligned}
$$

where we have made the usual approximation that $\partial W\left(1^{+}, 2\right) / \partial G(4,3) \approx 0$. Apparently, for consistency between the GWA and BSE, the $G W$ part of the MBPT should employ the identical screened Coulomb interaction as the BSE. This implies using exactly the same q-point grid for the internal summation leading to the self-energy, as well as employing exactly the same modified interaction $\tilde{W}(\mathbf{q})$. We have observed that by this procedure, excitons and their excitation energy converge rapidly with respect to reciprocalspace sampling. Examples are discussed below. If the abovementioned condition is violated, e.g., by using sparse q-point sampling in GWA (for efficiency sake) and fine sampling in the BSE, convergence with respect to reciprocal-space sampling can be significantly worse, simply because GWA and BSE employ different interactions $W$.

It should be noted again that the statements above are not restricted to the $2 \mathrm{D}$ materials discussed in this paper. All arguments concerning matching reciprocal-space samplings are equally valid for all kinds of condensed matter.

While the statements above hold for MBPT in general, there is one additional feature relevant for our present LDA $+G d W$ version of MBPT. As mentioned above, the term $-i G W_{\text {metal }}$ compensates the LDA exchange-correlation potential while $+i G W$ represents the self-energy. Following the discussion above, the latter term $+i G W$ must match the representation of the BSE and is therefore evaluated on the grid as indicated in Fig. 1(b), including the modified interaction $\tilde{W}$. The former term $-i G W_{\text {metal }}$, on the other hand, can be treated differently, following its own convergence behavior. It turns out that this term converges most rapidly when using $W_{\text {metal }}(\mathbf{q})$ on a grid avoiding $\mathbf{q}=0$, instead of a modified $\tilde{W}_{\text {metal }}$ like Eq. (11). The reason is given by the specific behavior of metallic screening, $W_{\text {metal }}(\mathbf{q}) \sim 1 /\left(q^{2}+q_{\mathrm{TF}}^{2}\right)$, which is qualitatively different from the semiconducting screening occurring in $\Sigma$ and in the BSE.

\section{E. Setting up the Bethe-Salpeter equation}

To obtain the excitonic properties, i.e., the absorption spectrum, four numerical steps are necessary: (i) a DFTLDA calculation, (ii) a LDA $+G d W$ calculation that yields the quasiparticle band structure energies $E_{n \mathbf{k}}^{\mathrm{QP}}$, (iii) evaluation of the electron-hole interaction kernel $K_{v c, v^{\prime} c^{\prime}}=-W_{v^{\prime} c, v c^{\prime}}+$ $V_{v^{\prime} c, c^{\prime} v}$ which enters into (iv) a BSE calculation where we set up and diagonalize the BSE-Hamilton operator with matrix elements

$$
\begin{aligned}
\left\langle v c\left|\hat{H}^{(e h)}\right| v^{\prime} c^{\prime}\right\rangle= & \left(E_{c}^{\mathrm{QP}}-E_{v}^{\mathrm{QP}}\right) \delta_{c c^{\prime}} \delta_{v v^{\prime}} \quad\left(\hat{H}_{\mathrm{BS}}\right) \\
& -\left(W_{v^{\prime} c, v c^{\prime}}-V_{v^{\prime} c, c^{\prime} v}\right) \quad\left(\hat{H}_{e h}\right) .
\end{aligned}
$$

In this notation, the indices $v$ and $c$ include the band $v(c)$ and the wave vector $\mathbf{k}$, e.g., $c=(c, k)$. Only the bands around the gap that are most important for the lowest-energy optical transitions are included in the BSE Hamiltonian [Eq. (12)]. The number of needed valence and conduction bands that are taken into account has to be checked by calculating the absorption spectrum for various included bands. In this study we typically employ four valence bands and six conduction bands (eight valence and eight conduction bands in the case of $\mathrm{ReSe}_{2}$ ).

Note that due to the requirements discussed in Sec. II D the matrix elements $W_{v^{\prime} c, v c^{\prime}}$ and $V_{v^{\prime} c, c^{\prime} v}$ are exactly the same as occurring in the $G W$ (or LDA $+G d W$ ) part of MBPT, which yields efficient numerical performance.

\section{CONVERGENCE DISCUSSION}

In this chapter we will discuss the numerical requirements and the convergence of the many-body calculation to evaluate electronic (Sec. III B) as well as optical properties (Sec. III D). Because the many-body calculation includes the description of long-range Coulomb interactions, one has to take special care of artificial interlayer interactions (see Sec. IIIC). We start with a very brief discussion of the initial DFT calculation, which is numerically extremely stable (Sec. III A). All calculations are carried out using codes written by ourselves [36,37]. 

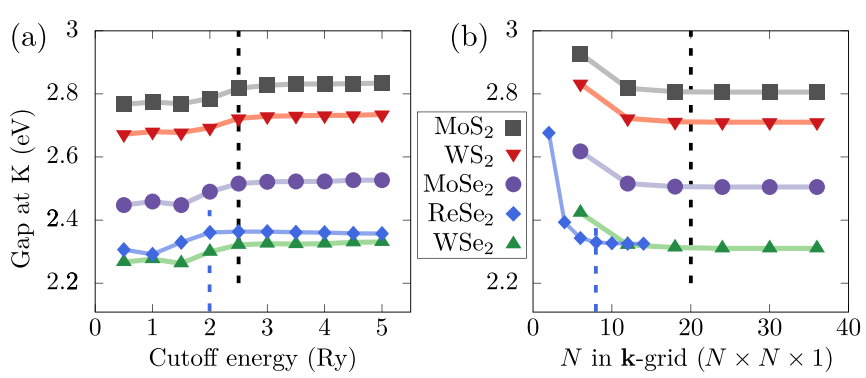

FIG. 2. (a) Convergence of the direct quasiparticle gap at the $K$ point (at $\Gamma$ for $\mathrm{ReSe}_{2}$ ) with respect to the energy cutoff in the auxiliary plane-wave basis for the given TMDCs. The $\mathbf{k}$-point grid is fixed at $20 \times 20 \times 1$ (at $8 \times 8 \times 1$ for $\mathrm{ReSe}_{2}$ ). (b) Convergence with respect to the k-point grid. The $\mathrm{LDA}+G d W$ basis is fixed at $2.5 \mathrm{Ry}$ (at $2 \mathrm{Ry}$ for $\mathrm{ReSe}_{2}$ ). All solid lines are a guide to the eye and the dashed lines indicate the applied cutoffs for the basis and the grid, respectively. The interlayer distance is always fixed at $45 \AA$ (at $56 \AA$ for $\mathrm{ReSe}_{2}$ ). All materials show similarly fast convergence behavior.

\section{A. DFT calculation}

The starting point for our many-body calculations is a DFT calculation carried out in the local density approximation (LDA), in the parametrization of Perdew and Zunger [38]. Norm-conserving pseudopotentials [39] in KleinmanBylander form [40] are used that also include spin-orbit interaction. We employ a basis of three shells of Gaussian orbitals with $s, p, d$, and $s^{*}$ symmetry per atom and decay constants between 0.13 and $2.50 a_{\mathrm{B}}^{-2}$ for all presented materials. For the group VI TMDCs with three atoms in the unit cell, this results in a basis size of 180 . For $\mathrm{ReSe}_{2}$ with 12 atoms in the unit cell, the size of the basis is 720 . In reciprocal space a k-point grid of $10 \times 10 \times 1$ is employed for these materials. All atomic positions are structurally relaxed until forces are smaller than $10^{-4} \frac{\mathrm{Ry}}{a_{\mathbf{B}}}$.

The theoretically optimized crystal structures are used in this work. We find lattice constants of 3.16, 3.30, 3.15, and $3.30 \AA$ for $\mathrm{MoS}_{2}, \mathrm{MoSe}_{2}, \mathrm{WS}_{2}$, and $\mathrm{WSe}_{2}$, which agree well with the experimental values of 3.160 [41], 3.299 [41], 3.155 [42], and 3.280 [43] Å. This shows the excellent results of LDA for the crystal structure containing covalent bonds within TMDCs. For $\mathrm{ReSe}_{2}$ we find a lattice constant $a$ of 6.61 $\AA$, which is in good agreement with the experimental value for bulk $\mathrm{ReSe}_{2}$ of $6.597 \AA$ [44].

\section{B. Electronic properties from $\mathrm{LDA}+G d W$}

In MBPT the existence of two-point functions requires a second, auxiliary basis. In our LDA $+G d W$ approach for the group VI TMDCs, we use plane waves with a cutoff of $2.5 \mathrm{Ry} \approx 34 \mathrm{eV}$ (2.0 Ry for $\mathrm{ReSe}_{2}$ ) which shows a convergence better than about $0.05 \mathrm{eV}$ as depicted in Fig. 2(a). Notably, conventional $G W$ calculations frequently report planewave cutoff energies of up to 35 Ry $[12,15,45]$ which is a factor of 14 larger. This clearly demonstrates the numerical efficiency of the LDA $+G d W$ method, whose much smaller auxiliary basis size is related to the similar asymptotic behavior of $W$ and $W_{\text {metal }}$ at large $\mathbf{G}$ (see the discussion in Ref. [21]).

Figure 2(b) shows the k-point grid convergence of the quasiparticle gap, which also shows very fast convergence.
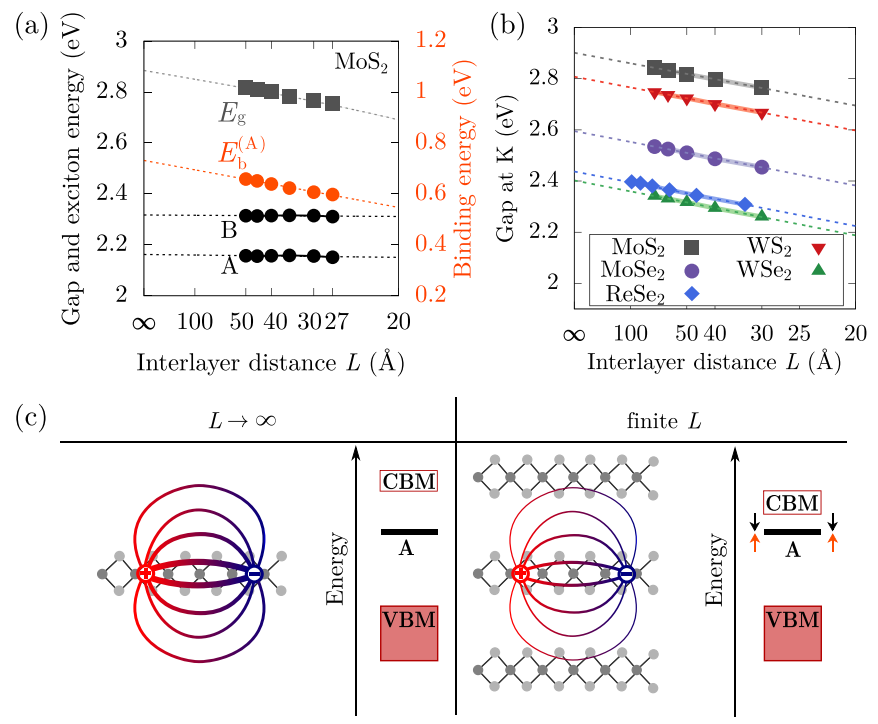

FIG. 3. (a) Band gap $E_{\mathrm{g}}$ (gray), exciton binding energy $E_{\mathrm{b}}^{(\mathrm{A})}$ (orange), and excitation energies of the $A$ and $B$ excitons (black) as a function of interlayer distance $L$. All dashed lines are linear fits. (b) Band gap at $K$ for different materials, revealing similar quantitative dependence on $L$. (c) Schematic representation of an isolated monolayer (left side) compared to a calculation with a small interlayer distance $L$ (right side). Depicted is an excited electron (red) hole (blue) pair bound through schematic field lines. At a finite $L$, the binding energy is reduced and at the same time, the gap is decreased, leaving the excitation energy $(A)$ unaffected. The involved energy positions are also sketched (not to scale).

Since the unit cell of $\mathrm{ReSe}_{2}$ is approximately twice as large as for the group VI TMDCs, the converged k-point grid is half as large.

One further important difference between $G W$ calculations on TMDC monolayers and our approach is the number of empty bands in the summation for the self-energy. Reported values for GWA are as high as $6000[12,15,45]$ where we use only 180 bands.

\section{Artificial interlayer interaction}

Although being two dimensional, the presently studied monolayers are periodically repeated in the third direction for practical purposes (employment of software requiring three-dimensional periodicity). In such a configuration, the dielectric response of the repeated monolayers leads to further screening of the screened Coulomb interaction within the monolayer in question, affecting the self-energy operator. This causes an (artificial) closing of the fundamental band gap depending on the interlayer distance (i.e., vertical lattice constant) $L$. Such behavior can be understood as an imagepotential effect, i.e., a charged quasiparticle in the monolayer (electron or hole) induces image charges in the other monolayers with which it interacts, affecting its energetics. In summary, the fundamental band gap behaves as $E_{g}(L)=$ $E_{g}(\infty)-\alpha / L$, as demonstrated in Fig. 3(a). In contrast to this single-particle behavior, an exciton within a monolayer shows no significant dependence on the interlayer distance $L$. This corresponds to the charge neutrality of an exciton. At 
most, its dipole moment can induce image dipole moments in the other monolayers. The resulting dipole-dipole interaction scales like $\sim 1 / L^{3}$, i.e., it decays rapidly with increasing $L$. Consequently, two-particle excitation energies of a monolayer converge rapidly with $L$, as illustrated in Fig. 3(a). We note in passing that DFT calculations do not incorporate any of these long-range screening effects and therefore do not exhibit band-gap renormalization due to neighboring material (neither the artificial effect on monolayers as discussed here, nor the real effect when monolayers are stacked towards multilayers or bulk crystals).

Figure 3(c) schematically compares two situations: an isolated $\mathrm{MoS}_{2}$ monolayer and a layer interacting with another one, i.e., for a moderate value of $L$. The excitation energy (A) is not shifted, while the band gap closes. Two larger effects cancel each other: the band gap $E_{g}$ closes [squares in Fig. 3(a)] and at the same time the binding energy $E_{b}^{(A)}$ is reduced [orange dots in Fig. 3(a)], leaving the excitation energies almost constant (black dots, labeled $A$ and $B$ ).

It is important to note that this fast convergence of excitonic energies with $L$ results from the exact matching of the k-point samplings in GWA and BSE (see again Sec. II D). Alternatively, truncation of the Coulomb interaction in the vertical direction has been suggested (see, e.g., Refs. [11,15]). However, Qiu et al. as well as Hüser et al. observe slower convergence with k-point sampling compared to our approach $[11,15]$. Rasmussen et al. observed that this unfavorable k-point convergence can be improved by exploiting the analytically known behavior of the screened Coulomb interaction in two dimensions [46]. In total, our findings suggest that exactly matching k-point sets as employed here, without trying to suppress interlayer interaction, also allows for a minimum number of $\mathbf{k}$ points to be used. Furthermore, including three-dimensional Coulomb interaction and screening also allows to study the (real) interaction of electrons, holes, and excitons with substrates, adsorbates, and multilayer systems, which might be much more complicated when a truncated interaction is used.

Although our main interest is the evaluation of excitonic states, the precise determination of the fundamental band gap of a monolayer is also relevant. To achieve this, the band gap as shown in Figs. 3(a) and 3(b) is evaluated for several values of $L$, followed by (linear) extrapolation in $1 / L$ to $L \rightarrow \infty$.

\section{Optical properties}

Figure 4(a) shows the absorption spectrum of a $\mathrm{MoS}_{2}$ monolayer depending on the number of bands in the BSE Hamiltonian. The spectra show a distinct convergence behavior, in particular at higher energies. For example, to accurately describe the $C$ exciton, at least four valence and six conduction bands have to be taken into account. For the $A$ and $B$ excitons, two valence and two conduction bands are already sufficient for a qualitative picture, but the excitation energy is better described by two valence and four conduction bands. If the highest valence band only is taken into account (see graph labeled $1 / 2$ ), the $B$ and $B_{2 s}$ excitons vanish and the spectrum below $2.7 \mathrm{eV}$ is dominated by the $A$ and $A_{2 s}$ excitations. The absorption spectra of monolayer $\mathrm{ReSe}_{2}$ for various included bands is shown in Fig. 4(d). Since the unit cell of $\mathrm{ReSe}_{2}$ contains four times as many atoms as a unit cell of a group
VI TMDC, many more bands are needed for an equally good convergence, as can be seen in the figure.

Figure 4(b) shows the convergence of the BSE spectrum of a $\mathrm{MoS}_{2}$ monolayer as a function of the k-point grid density (see Sec. IIE) which shows a fast convergence with little visible differences for the $A$ and $B$ excitons. Convergence of the excitation energies with respect to the $\mathbf{k}$-point grid is quantified in Fig. 4(c) for the $A, B$, and excited $A_{2 s}$ states. Importantly, for $A$ and $B$ all calculated values between a $9 \times$ $9 \times 1$ grid and the extrapolations for $N \rightarrow \infty$ in $N \times N \times 1$ lie within an extremely narrow energy window of $\sim 0.1 \mathrm{eV}$, and the extrapolation to $N \rightarrow \infty$ appears numerically reliable to within $0.01 \mathrm{eV}$. The excited $A_{2 s}$ state converges a bit slower due to the more complicated structure of the wave function with two local maxima and a larger extent in real space [12]. Note that the $A$ exciton is already converged better than $0.05 \mathrm{eV}$ at grids as small as $15 \times 15 \times 1$, which we find equivalently for the $B$ exciton. This extremely fast convergence results from a combination of several aspects to our approach. In particular, each of these BSE calculations starts from a corresponding LDA $+G d W$ band structure calculation employing the same respective k-point sampling. The $\mathbf{k}$-point grids for the internal summation in the self-energy operator are also chosen exactly equivalent to the grids for the quasiparticle corrections. With such exactly matching grids, the LDA $+G d W$ (as well as $G W$ ) quasiparticle gap and the electron-hole interaction show the same asymptotic behavior with increasing grid density, and the exciton energy (i.e., the combination of both) converges rapidly with the number of $\mathbf{k}$ points. Additionally, the Coulomb interaction between adjacent supercells is not truncated, which also reduces the convergence requirements [11]. A similar behavior can be found for absorption spectra of monolayer $\mathrm{ReSe}_{2}$ with different kpoint grids in Fig. 4(e). As it is shown in Fig. 4(f), already for a k-point grid of $9 \times 9 \times 1$ the excitation energies of $X_{1}$, $X_{4}$, and $X_{5}$ are converged better than $0.05 \mathrm{eV}$, if compared to the extrapolation to $N \rightarrow \infty$. Note that the relative energies (among the group $X_{1}-X_{5}$ ) are converged even much better.

We note that other numerical approaches often report larger k-point samplings of up to $300 \times 300 \times 1$ [12,47]. In this work, the fast convergence of the excitation energies with respect to the $\mathbf{k}$-point sampling results from the following issues:

(i) Identical grids for the band structure and electron-hole $\mathbf{k}$ points.

(ii) Identical k-point grids for the internal summation in the self-energy and for the electron-hole interaction matrix elements.

(iii) No truncation of the Coulomb interaction.

\section{RESULTS}

\section{A. Quasiparticle band structures of $\mathrm{MoS}_{2}, \mathrm{MoSe}_{2}$, $\mathrm{WS}_{2}, \mathrm{WSe}_{2}$, and $\mathrm{ReSe}_{2}$ monolayers}

Figure 5(a) shows the effect on the band structure of a $\mathrm{MoS}_{2}$ monolayer when going from LDA (dashed black bands) to the converged LDA $+G d W$ (black and colored bands). We find a large increase in the band gap from $1.78 \mathrm{eV}$ in LDA to $2.90 \mathrm{eV}$ in $\mathrm{LDA}+G d W$ [extrapolated to $L \rightarrow \infty$, see Fig. 3(a)]. The magnitude of the spin-orbit splitting in the valence band at $K$ is increased from 150 to $180 \mathrm{meV}$ and the 

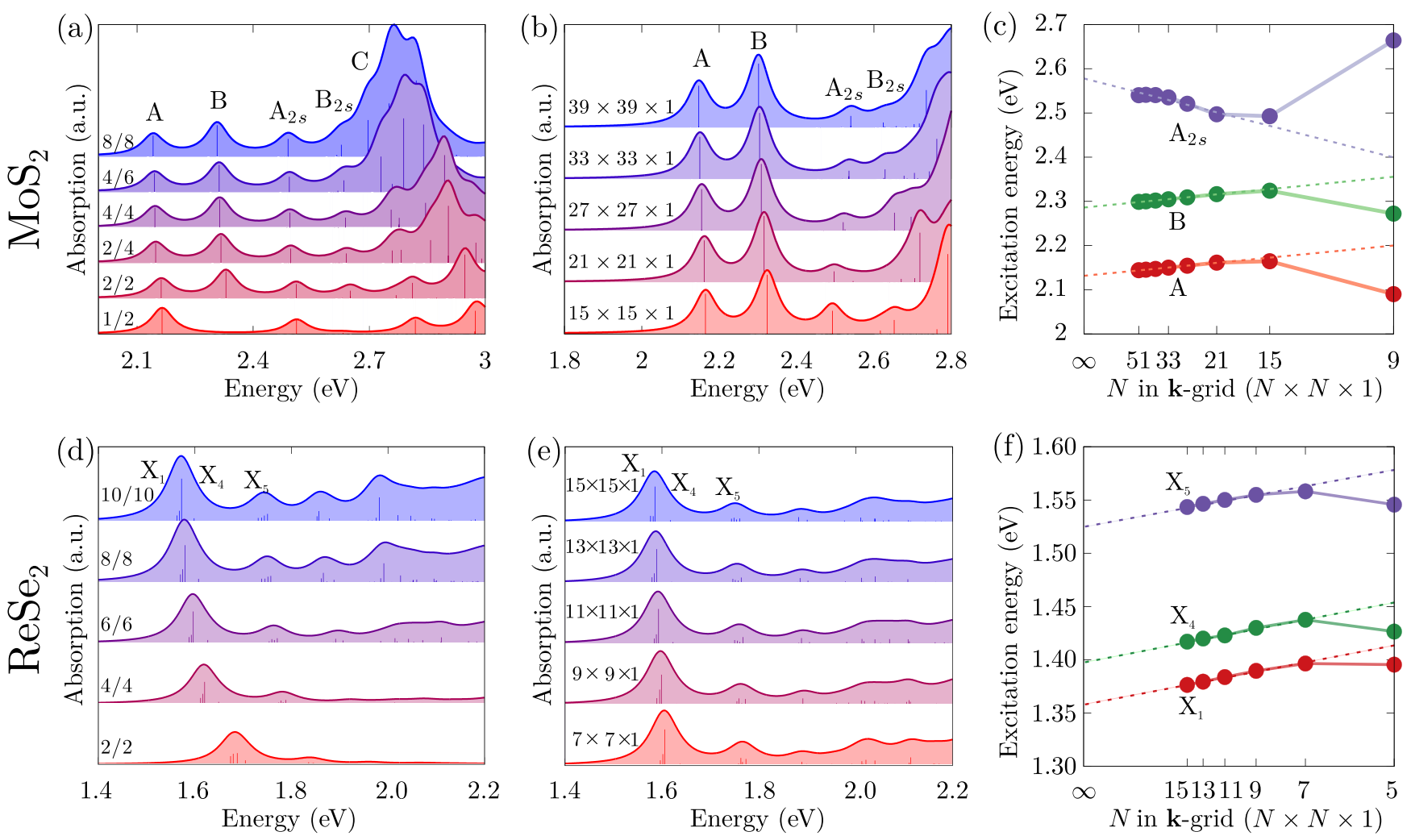

FIG. 4. (a) BSE absorption spectrum of a $\mathrm{MoS}_{2}$ monolayer with different numbers of valence (first number) and conduction bands (second number) included in the BSE Hamiltonian. A fixed k-point grid of $10 \times 10 \times 1$ is used. (b) Convergence of the BSE absorption spectrum of a $\mathrm{MoS}_{2}$ monolayer as function of the k-point grid with two valence and four conduction bands included. A vertical offset is applied in (a) and (b) for improved visibility. (c) Excitation energies for the three lowest excitations of a $\mathrm{MoS}_{2}$ monolayer as a function of the k-point grid. The dashed lines are a linear fit applied for $N \geqslant 21$. Note that the $A_{2 s}$ exciton converges slightly slower. (d) BSE absorption spectrum of a ReSe ${ }_{2}$ monolayer with different numbers of valence (first number) and conduction bands (second number) included in the BSE Hamiltonian. A fixed k-point grid of $11 \times 11 \times 1$ is used. (e) Convergence of the BSE absorption spectrum of a $\operatorname{ReSe}_{2}$ monolayer as function of the k-point grid with eight valence and eight conduction bands included. A vertical offset is applied in (d) and (e) for improved visibility. (f) Excitation energies of three chosen excitations of a $\mathrm{ReSe}_{2}$ monolayer as a function of the k-point grid. For all spectra throughout this work, the wave vector of the photon is chosen perpendicular to the monolayer with linearly polarized light within the plane.

splitting of the two lowest conduction bands at $K$ is increased as well from merely $\sim 2 \mathrm{meV}$ in LDA to $\sim 15 \mathrm{meV}$ in the quasiparticle picture. Another important difference in going from LDA to LDA $+G d W$ is the decrease of the effective masses in the valence-band maximum from 0.60 to 0.51 and in the conduction-band minimum from 0.56 to 0.47 (all in units of the electron mass).

Even though $\mathrm{MoS}_{2}$ has a special role among TMDCs, research has quickly spread to other members of this group [48]. Three further widely studied systems are $\mathrm{MoSe}_{2}, \mathrm{WS}_{2}$, and $\mathrm{WSe}_{2}$ that are shown in Figs. 5(b)-5(d).

The similarities in the crystal structure of the four TMDCs are reflected in the band structures that all show analog features. All materials have the lowest direct transition at the $K$ points both in LDA and LDA $+G d W$ with spin-split bands both in the valence and conduction bands. For $\mathrm{WS}_{2}$ and $\mathrm{WSe}_{2}$ the lowest conduction band at $K$ and in the minimum along $\overline{K \Gamma}$ are energetically very close together so that the gap is slightly indirect as discussed for example by Selig et al. [49]. We stress that the relative position of these two minima of the conduction band sensitively depends on the underlying lattice structure (see, e.g., the discussion by Conley et al. [50] and by Steinhoff et al. [51]).

We find quasiparticle band gaps of 2.90, 2.60, 2.81, and $2.40 \mathrm{eV}$ for $\mathrm{MoS}_{2}, \mathrm{MoSe}_{2}, \mathrm{WS}_{2}$, and $\mathrm{WSe}_{2}$. The size of the spin-orbit splitting of the highest valence bands at $K$ follows $\mathrm{MoS}_{2}<\mathrm{MoSe}_{2}<\mathrm{WS}_{2}<\mathrm{WSe}_{2}$ with values of 180, 230, 510 , and $570 \mathrm{meV}$. It is determined by the atomic number (with stronger SOC in heavier atoms [52]), following $\mathrm{Mo}<$ $W$ and $\mathrm{S}<\mathrm{Se}$.

The spin splitting in the lowest conduction bands at $K$ is much smaller with values of $15,42,-10$, and $-7 \mathrm{meV}$ for $\mathrm{MoS}_{2}, \mathrm{MoSe}_{2}, \mathrm{WS}_{2}$, and $\mathrm{WSe}_{2}$ but shows an interesting feature: the spin character of the two conduction bands is reversed in the Mo compounds as compared to the $W$ compounds. For $\mathrm{MoS}_{2}$ and $\mathrm{MoSe}_{2}$, this inversion leads to a crossing of the lowest conduction bands around $K$, visible in the insets of Fig. 5. The ordering of the conduction bands is important for the optical properties since excitations at the $K$ point leave the spin invariant.

The presented group VI TMDCs possess a high inplane symmetry, rendering them unfavorable for the study of 

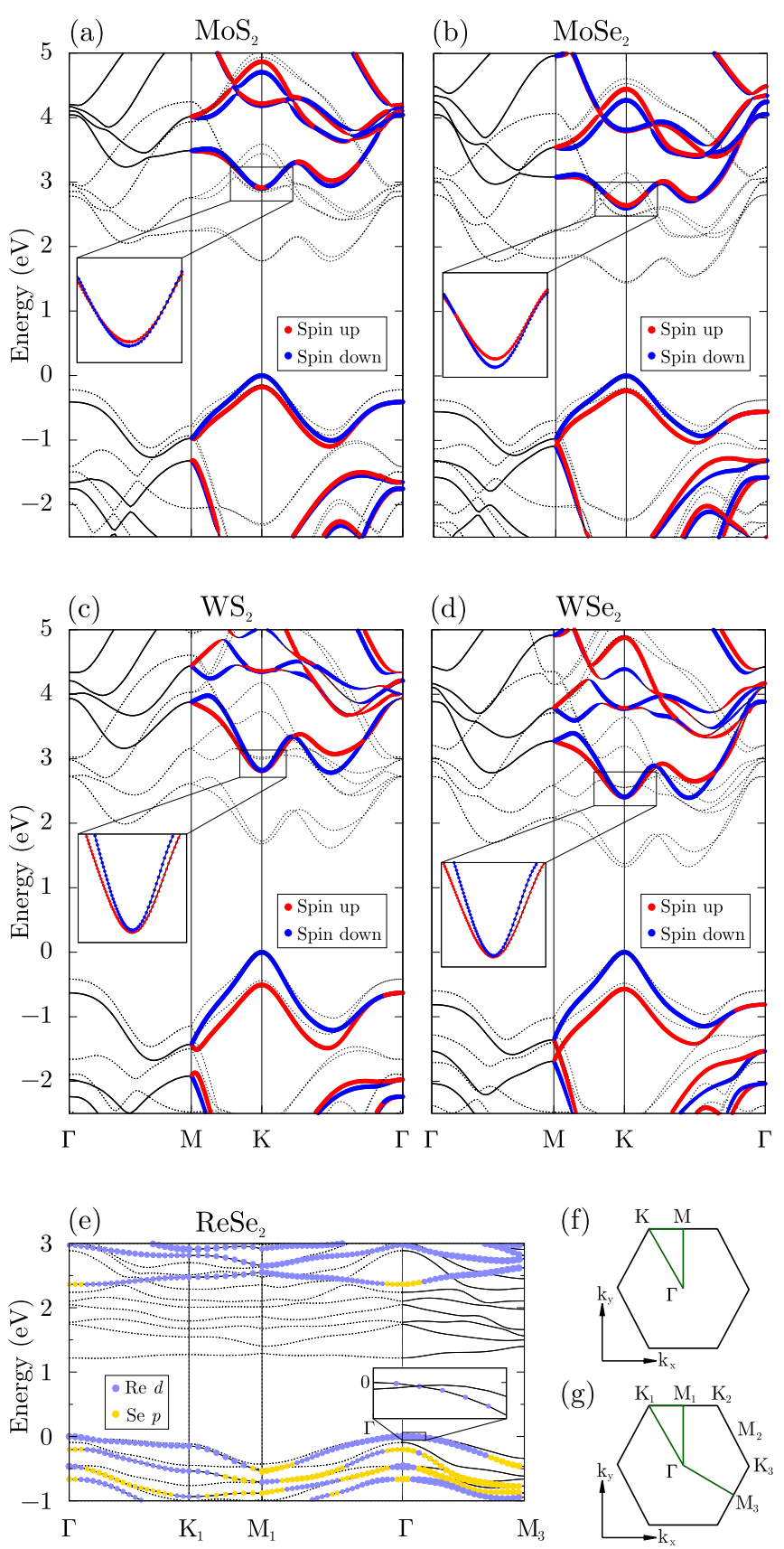

FIG. 5. (a)-(d) Comparison of the quasiparticle $(\mathrm{LDA}+G d W$, black and colored) and LDA (dashed gray) band structures of $\mathrm{MoS}_{2}$, $\mathrm{MoSe}_{2}, \mathrm{WS}_{2}$, and $\mathrm{WSe}_{2}$ monolayer. The size of the added points is proportional to the expectation value of the spin operator in the direction perpendicular to the monolayer (spin up in red, spin down in blue). The insets show the spin-split conduction bands at $K$ with an inverted ordering between Mo and $W$ based TMDCs. The k-point grid in the quasiparticle calculations is fixed at $20 \times 20 \times 1$ points. (e) Comparison of the quasiparticle ( $\mathrm{LDA}+G d W$, black and colored) and LDA (dashed gray) band structures of $\mathrm{ReSe}_{2}$ monolayer. The size of the added points is proportional to the most prominent orbital contribution to the state ( $\operatorname{Re} d$ orbitals in blue, Se $p$ orbitals in yellow). The k-point grid in the quasiparticle calculations is fixed at $8 \times 8 \times 1$ points. For further numerical details we refer to the main text. (f) Brillouin zone of $\mathrm{MoS}_{2}, \mathrm{MoSe}_{2}, \mathrm{WS}_{2}$, and $\mathrm{WSe}_{2}$. (g) Brillouin zone of $\mathrm{ReSe}_{2}$, which slightly deviates from a perfect hexagon [53].

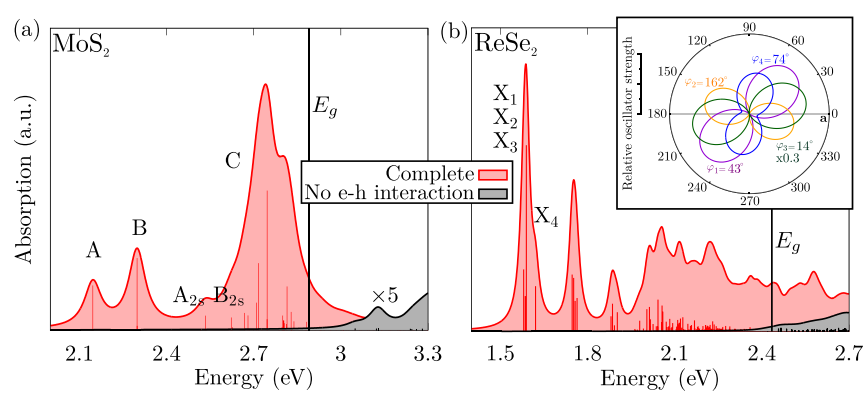

FIG. 6. (a) Exciton absorption spectrum (red) of a $\mathrm{MoS}_{2}$ monolayer for vertically incident light. Also shown is the free absorption spectrum without electron-hole interaction (black, multiplied by five). The quasiparticle gap $E_{g}$ is indicated at $2.90 \mathrm{eV}$. A k-point grid of $33 \times 33 \times 1$, four valence and six conduction bands, as well as an interlayer distance of $45 \AA$ are used. At every bright excitation (see red and black straight lines) an artificial Lorentzian function with a width of $0.035 \mathrm{eV}$ is applied to simulate the broadening in experimental measurements. (b) Unpolarized exciton absorption spectrum (red) of a $\mathrm{ReSe}_{2}$ monolayer. Also shown is the free absorption spectrum without electron-hole interaction (black). The quasiparticle gap $E_{g}$ is indicated at $2.44 \mathrm{eV}$. A k-point grid of $11 \times 11 \times 1$, eight valence and eight conduction bands, as well as an interlayer distance of $56 \AA$ are used (Lorentzian broadening $0.015 \mathrm{eV}$ ). The inset shows the response of the excitons to linearly polarized light with different angles of incident.

anisotropic effects. Therefore, we also investigated monolayer $\mathrm{ReSe}_{2}$ which is another member of the TMDC family. It crystallizes in a $1 T^{\prime}$ structure leading to a reduced symmetry of the crystal [53]. Four Re atoms form a parallelogramlike shape which leads to so-called "rhenium-diamond-chains" along the crystallographic $a$ axis (for details see [44]). Figure 5(g) shows the Brillouin zone and the green lines indicate the path through the Brillouin zone along which the band structure is shown in Fig. 5(e). The points $K_{1}, K_{2}$, and $K_{3}$ (as well as $M_{1}, M_{2}$, and $M_{3}$ ) are not equivalent. In contrast to the other TMDCs, $\mathrm{ReSe}_{2}$ is inversion symmetric and each band is twofold degenerate (i.e., no spin polarization).

Going from LDA to LDA $+G d W$, the band gap of monolayer $\mathrm{ReSe}_{2}$ opens up from 1.22 to $2.44 \mathrm{eV}$. This is in very good agreement with another theoretical work [54], which found a band gap of $2.29 \mathrm{eV}$ from a conventional $G W$ calculation. The anisotropy of the crystal structure is clearly visible in the band structure, e.g., when looking at the different $\Gamma-M$ directions in Fig. 5(e). It is important to note that the change from LDA to $\mathrm{LDA}+G d W$ also changes the location of the valence band maximum. This leads to a change in the nature of the band gap from indirect in LDA to direct in LDA $+G d W$. The same change from indirect to direct was also found by Zhong et al. within conventional GWA [54].

\section{B. Optical properties}

Figure 6(a) shows exemplarily the calculated optical spectrum of a $\mathrm{MoS}_{2}$ monolayer. The black line (amplified by a factor of 5) shows the absorption spectrum without electron-hole interactions. Here, only direct interband transitions contribute; it thus starts at the direct quasiparticle band gap $E_{g}$. 
Strikingly, the absorption spectrum including electron-hole interactions is entirely dominated in the low-energy range by excitons, which are commonly denoted as $A$ and $B$ (at low energy), as well as $C$ (a broader absorption feature composed of several lines, which is often found slightly below the fundamental band gap) $[15,55]$. The two lowest transitions labeled $A$ at $2.15 \mathrm{eV}$ and $B$ at $2.30 \mathrm{eV}$ are direct transitions from the spin-split valence band at $K$ into the lowest conduction band. Therefore, their energetic distance $(150 \mathrm{meV})$ mainly corresponds to the spin-orbit splitting of the involved valence bands $(180 \mathrm{meV})$. The corresponding excited states $A_{2 s}$ and $B_{2 s}$ are found at higher energies. These excitations stem from the same interband transitions as $A$ and $B$, but are $2 s$-like higher excited states of the electron-hole pair. The excited states of excitons in 3D often approximately follow energy series known from the hydrogen atom. Excitons in 2D, however, do not follow the corresponding " $2 \mathrm{D}$ hydrogen" energy series since different excited states experience different effective screenings [56,57]. In conventional 3D semiconductors like GaAs, exciton binding energies are in the order of millielectron volt $[58,59]$. In the $3 \mathrm{D}$ crystals of the TMDC materials, exciton binding energies amount to less than about $200 \mathrm{meV}$. Going from 3D to 2D, screening is drastically reduced, which in turn results in an increased binding energy of $740 \mathrm{meV}$ for the $A$ exciton in our calculation. This value fits well to numbers reported in the literature that lie between 0.5 and $1 \mathrm{eV}$ using similar techniques [3-6]. At about $2.7 \mathrm{eV}$, the $C$ exciton is located [Fig. 6(a)]. It consists of several different transitions mainly situated near the $K$ point and approximately halfway along the direction $K-\Gamma$ (at the local minimum of the lowest conduction band).

Figure 6(b) shows the calculated absorption spectra of monolayer $\mathrm{ReSe}_{2}$. Due to the electron-hole interaction, strongly bound excitons are observed compared to transitions excluding the electron-hole interactions (black). In the lowenergy part of the spectrum we find groups of four excitations with a small energy separation. These four transitions stem from the degenerate bands (e.g., two valence and two conduction bands allow four transitions). The two spin orientations of each pair of degenerate bands lead to different spin combinations of electron and hole in the four excitons. This results in different contributions of the exchange part to the BSE Hamiltonian which induces a small energetic splitting of such four excitons. For the energetically lowest exciton $X_{1}$ in a $\operatorname{ReSe}_{2}$ monolayer we find an excitation energy of $1.58 \mathrm{eV}$ resulting in an excitonic binding energy of $0.86 \mathrm{eV}$.

Since both crystal structure and band structure of $\mathrm{ReSe}_{2}$ show in-plane anisotropy, also the excitons should reflect that. In the inset of Fig. 6(b) we show the optical oscillator strength of the first four excitons of monolayer $\mathrm{ReSe}_{2}$. Here, the incident light is perpendicular to the layer. All excitations exhibit a distinct dipole character with different orientations. Both energetic positions and polarizations of these excitons are in good agreement with experimental results [31].

Table II summarizes all our data for low-energy excitons in the five studied materials. For comparison, the last column summarizes available $G W / \mathrm{BSE}$ results for the lowest exciton from the literature [3,54,60-62]. For the conventional TMDCs $\left(\mathrm{MoS}_{2}, \mathrm{MoSe}_{2}, \mathrm{WS}_{2}\right.$, and $\left.\mathrm{WSe}_{2}\right)$, this incomplete list focuses
TABLE II. Summary of theoretical results from our LDA $+G d W /$ BSE calculations: Gap $E_{g}, A$ and $B\left(X_{1}\right.$ and $\left.X_{5}\right)$ excitation energies, their energetic splitting $\Delta$, and their binding energies $E^{b}$. The gap is determined using $20 \times 20 \times 1 \mathbf{k}$ points and an extrapolation for $L \rightarrow \infty$. For comparison, the last column summarizes $G W / \mathrm{BSE}$ results for the lowest exciton from the literature [3,54,60-62].

\begin{tabular}{lccccccc}
\hline \hline$(\mathrm{eV})$ & $E_{g}$ & $A$ & $B$ & $\Delta_{A B}$ & $E_{A}^{b}$ & $E_{B}^{b}$ & $A(G W / \mathrm{BSE})$ \\
\hline $\mathrm{MoS}_{2}$ & 2.90 & 2.15 & 2.30 & 0.15 & 0.74 & 0.60 & $1.78^{\mathrm{a}}, 1.82^{\mathrm{b}}, 2.00^{\mathrm{c}}$ \\
$\mathrm{MoSe}_{2}$ & 2.60 & 1.78 & 2.00 & 0.21 & 0.81 & 0.60 & $1.50^{\mathrm{a}}, 1.67^{\mathrm{b}}, 1.62^{\mathrm{c}}$ \\
$\mathrm{WS}_{2}$ & 2.81 & 2.20 & 2.62 & 0.42 & 0.61 & 0.19 & $1.84^{\mathrm{a}}, 2.152^{\mathrm{b}}, 2.07^{\mathrm{c}}$ \\
$\mathrm{WSe}_{2}$ & 2.40 & 1.78 & 2.24 & 0.45 & 0.62 & 0.16 & $1.52^{\mathrm{a}}, 1.75^{\mathrm{b}}, 1.71^{\mathrm{c}}$ \\
$(\mathrm{eV})$ & $E_{g}$ & $X_{1}$ & $X_{5}$ & $\Delta_{X_{1} X_{5}}$ & $E_{X_{1}}^{b}$ & $E_{X_{2}}^{b}$ & $X_{1}(G W / \mathrm{BSE})$ \\
\hline $\mathrm{ReSe}_{2}$ & 2.44 & 1.58 & 1.75 & 0.17 & 0.86 & 0.69 & $1.42^{\mathrm{d}}, 1.43^{\mathrm{e}}$ \\
\hline \hline
\end{tabular}

${ }^{\mathrm{a}}$ Reference [3].

${ }^{\mathrm{b}}$ Reference [60].

${ }^{\mathrm{c}}$ Reference [61].

${ }^{\mathrm{d}}$ Reference [54].

${ }^{\mathrm{e}}$ Reference [62].

on studies in which all four materials were investigated. The data exhibit some variation from study to study, as was already discussed in the Introduction. We also note that our present data are systematically higher in energy by some $0.1 \mathrm{eV}$ in the current implementation of our simplified LDA $+G d W / \mathrm{BSE}$ approach.

\section{CONCLUSIONS}

We have used many-body perturbation theory within the LDA $+G d W / \mathrm{BSE}$ approximation to describe the electronic and optical properties of five exemplary transition metal dichalcogenide monolayers. After recapitulating its theoretical background, we have discussed the fast convergence and numerical efficiency of the introduced implementation. Very accurate electronic and optical results have been observed at a fraction of the numerical cost of $G W / \mathrm{BSE}$ calculations. In particular, we employ reciprocal-space sampling grids that exactly match each other. The resulting convergence behavior allows to use relatively coarse grids without loss of accuracy. This issue holds for $G W / \mathrm{BSE}$ as well as for our simplified LDA $+G d W /$ BSE version. The numerical benefits mark $\mathrm{LDA}+G d W / \mathrm{BSE}$ as a good candidate for further theoretical investigations in the large growing field of two-dimensional materials.

\section{ACKNOWLEDGMENTS}

This work has been supported by the German Research Foundation (DFG) in terms of the Collaborative Research Centre SFB 1083. T.D. acknowledges financial support from the Villum foundation. We gratefully acknowledge the computing time granted by the John von Neumann Institute for Computing (NIC) and provided on the supercomputer JURECA at Jülich Supercomputing Centre (JSC). 
[1] K. F. Mak, C. Lee, J. Hone, J. Shan, and T. F. Heinz, Atomically Thin $\mathrm{MoS}_{2}$ : A New Direct-Gap Semiconductor, Phys. Rev. Lett. 105, 136805 (2010).

[2] A. Splendiani, L. Sun, Y. Zhang, T. Li, J. Kim, C.-Y. Chim, G. Galli, and F. Wang, Emerging photoluminescence in monolayer $\mathrm{MoS}_{2}$, Nano. Lett. 10, 1271 (2010).

[3] A. Ramasubramaniam, Large excitonic effects in monolayers of molybdenum and tungsten dichalcogenides, Phys. Rev. B 86, 115409 (2012).

[4] H.-P. Komsa and A. V. Krasheninnikov, Effects of confinement and environment on the electronic structure and exciton binding energy of $\mathrm{MoS}_{2}$ from first principles, Phys. Rev. B 86, 241201 (2012).

[5] J. Feng, X. Qian, C.-W. Huang, and J. Li, Strain-engineered artificial atom as a broad-spectrum solar energy funnel, Nat. Photonics 6, 866 (2012).

[6] I. Ben Amara, E. Ben Salem, and S. Jaziri, Optoelectronic response and excitonic properties of monolayer $\mathrm{MoS}_{2}$, J. Appl. Phys. 120, 051707 (2016).

[7] H. Zeng, J. Dai, W. Yao, D. Xiao, and X. Cui, Valley polarization in $\mathrm{MoS}_{2}$ monolayers by optical pumping, Nat. Nanotechnol. 7, 490 (2012).

[8] K. F. Mak, K. He, J. Shan, and T. F. Heinz, Control of valley polarization in monolayer $\mathrm{MoS}_{2}$ by optical helicity, Nat. Nanotechnol. 7, 494 (2012).

[9] G. Onida, L. Reining, and A. Rubio, Electronic excitations: Density-functional versus many-body Green's-function approaches, Rev. Mod. Phys. 74, 601 (2002).

[10] M. Rohlfing and S. G. Louie, Electron-hole excitations and optical spectra from first principles, Phys. Rev. B 62, 4927 (2000).

[11] F. Hüser, T. Olsen, and K. S. Thygesen, How dielectric screening in two-dimensional crystals affects the convergence of excited-state calculations: Monolayer $\mathrm{MoS}_{2}$, Phys. Rev. B 88, 245309 (2013).

[12] D. Y. Qiu, F. H. da Jornada, and S. G. Louie, Screening and many-body effects in two-dimensional crystals: Monolayer $M o S_{2}$, Phys. Rev. B 93, 235435 (2016).

[13] H. Shi, H. Pan, Y.-W. Zhang, and B. I. Yakobson, Quasiparticle band structures and optical properties of strained monolayer $\mathrm{MoS}_{2}$ and $\mathrm{WS}_{2}$, Phys. Rev. B 87, 155304 (2013).

[14] M. Bernardi, M. Palummo, and J. C. Grossman, Extraordinary sunlight absorption and one nanometer thick photovoltaics using two-dimensional monolayer materials, Nano Lett. 13, 3664 (2013).

[15] D. Y. Qiu, H. Felipe, and S. G. Louie, Optical Spectrum of $\mathrm{MoS}_{2}$ : Many-Body Effects and Diversity of Exciton States, Phys. Rev. Lett. 111, 216805 (2013).

[16] A. Molina-Sánchez, D. Sangalli, K. Hummer, A. Marini, and L. Wirtz, Effect of spin-orbit interaction on the optical spectra of single-layer, double-layer, and bulk $\mathrm{MoS}_{2}$, Phys. Rev. B 88, 045412 (2013).

[17] T. Cheiwchanchamnangij and W. R. L. Lambrecht, Quasiparticle band structure calculation of monolayer, bilayer, and bulk $\mathrm{MoS}_{2}$, Phys. Rev. B 85, 205302 (2012).

[18] C. Ataca and S. Ciraci, Functionalization of single-layer $\mathrm{MoS}_{2}$ honeycomb structures, J. Phys. Chem. C 115, 13303 (2011).

[19] R. S. Sundaram, M. Engel, A. Lombardo, R. Krupke, A. C. Ferrari, Ph. Avouris, and M. Steiner, Electroluminescence in Single Layer $\mathrm{MoS}_{2}$, Nano Lett. 13, 1416 (2013).
[20] S. W. Han, H. Kwon, S. K. Kim, S. Ryu, W. S. Yun, D. H. Kim, J. H. Hwang, J.-S. Kang, J. Baik, H. J. Shin, and S. C. Hong, Band-gap transition induced by interlayer van der Waals interaction in $\mathrm{MoS}_{2}$, Phys. Rev. B 84, 045409 (2011).

[21] M. Rohlfing, Electronic excitations from a perturbative LDA + GdW approach, Phys. Rev. B 82, 205127 (2010).

[22] L. Hedin, New method for calculating the one-particle green's function with application to the electron-gas problem, Phys. Rev. 139, A796 (1965).

[23] M. S. Hybertsen and S. G. Louie, Electron correlation in semiconductors and insulators: Band gaps and quasiparticle energies, Phys. Rev. B 34, 5390 (1986).

[24] M. Rohlfing, P. Krüger, and J. Pollmann, Efficient scheme for $G W$ quasiparticle band-structure calculations with applications to bulk $\mathrm{Si}$ and to the $\mathrm{Si}(001)-(2 \times 1)$ surface, Phys. Rev. B 52, 1905 (1995).

[25] J. Gesenhues, D. Nabok, M. Rohlfing, and C. Draxl, Analytical representation of dynamical quantities in $g w$ from a matrix resolvent, Phys. Rev. B 96, 245124 (2017).

[26] C. S. Wang and W. E. Pickett, Density-Functional Theory of Excitation Spectra of Semiconductors: Application to Si, Phys. Rev. Lett. 51, 597 (1983).

[27] F. Gygi and A. Baldereschi, Quasiparticle Energies in Semiconductors: Self-Energy Correction to the Local-Density Approximation, Phys. Rev. Lett. 62, 2160 (1989).

[28] V. Fiorentini and A. Baldereschi, Dielectric scaling of the selfenergy scissor operator in semiconductors and insulators, Phys. Rev. B 51, 17196 (1995).

[29] T. Deilmann, Structural, electronic and optical properties of organic adsorbates and further nanomaterials, Dissertation, Westfälische Wilhelms-Universität Münster, 2015.

[30] R. Schmidt, I. Niehues, R. Schneider, M. Drüppel, T. Deilmann, M. Rohlfing, S. M. de Vasconcellos, A. Castellanos-Gomez, and R. Bratschitsch, Reversible uniaxial strain tuning in atomically thin $\mathrm{WSe}_{2}$, 2D Mater. 3, 021011 (2016).

[31] A. Arora, J. Noky, M. Drüppel, B. Jariwala, T. Deilmann, R. Schneider, R. Schmidt, O. Del Pozo-Zamudio, T. Stiehm, A. Bhattacharya, P. Krüger, S. Michaelis de Vasconcellos, M. Rohlfing, and R. Bratschitsch, Highly Anisotropic in-Plane Excitons in Atomically Thin and Bulklike 1T'-ReSe 2 , Nano Lett. 17, 3202 (2017).

[32] R. Frisenda, M. Drüppel, R. Schmidt, S. Michaelis de Vasconcellos, D. Perez de Lara, R. Bratschitsch, M. Rohlfing, and A. Castellanos-Gomez, Biaxial strain tuning of the optical properties of single-layer transition metal dichalcogenides, 2D Mater. Applicat. 1, 10 (2017).

[33] M. Drüppel, T. Deilmann, P. Krüger, and M. Rohlfing, Diversity of trion states and substrate effects in the optical properties of an $\mathrm{MoS}_{2}$ monolayer, Nat. Commun. 8, 2117 (2017).

[34] P. Eickholt, J. Noky, E. F. Schwier, K. Shimada, K. Miyamoto, T. Okuda, C. Datzer, M. Drüppel, P. Krüger, M. Rohlfing, and M. Donath, Location of the valence band maximum in the band structure of anisotropic 1T'- $\operatorname{ReSe}_{2}$, Phys. Rev. B 97, 165130 (2018).

[35] F. Bechstedt, R. Enderleln, and R. Wischnewski, Binding energies and chemical shifts of least bound core electron excitations in cubic $\mathrm{A}_{N} \mathrm{~B}_{8-N}$ semiconductors, Phys. Status Solidi B 107, 637 (1981).

[36] J. Wieferink, P. Krüger, and J. Pollmann, Improved Hybrid Algorithm with Gaussian Basis Sets and Plane Waves: 
First-principles calculations of ethylene adsorption on $\beta$ SiC(001)-(3 × 2), Phys. Rev. B 74, 205311 (2006).

[37] M. Rohlfing, P. Krüger, and J. Pollmann, Quasiparticle bandstructure calculations for $\mathrm{C}, \mathrm{Si}, \mathrm{Ge}, \mathrm{GaAs}$, and $\mathrm{SiC}$ using Gaussian-orbital basis sets, Phys. Rev. B 48, 17791 (1993).

[38] J. P. Perdew and A. Zunger, Self-interaction correction to density-functional approximations for many-electron systems, Phys. Rev. B 23, 5048 (1981).

[39] D. R. Hamann, Generalized norm-conserving pseudopotentials, Phys. Rev. B 40, 2980 (1989).

[40] L. Kleinman and D. M. Bylander, Efficacious Form for Model Pseudopotentials, Phys. Rev. Lett. 48, 1425 (1982).

[41] Th. Böker, R. Severin, A. Müller, C. Janowitz, R. Manzke, D. Voß, P. Krüger, A. Mazur, and J. Pollmann, Band structure of $\mathrm{MoS}_{2}, \mathrm{MoSe}_{2}$, and $\alpha-\mathrm{MoTe}_{2}$ : Angle-resolved photoelectron spectroscopy and ab initio calculations, Phys. Rev. B 64, 235305 (2001).

[42] W. S. Yun, S. W. Han, S. C. Hong, I. G. Kim, and J. D. Lee, Thickness and strain effects on electronic structures of transition metal dichalcogenides: $2 \mathrm{H}-M X_{2}$ semiconductors $(M=$ Mo, W; $X=\mathrm{S}, \mathrm{Se}, \mathrm{Te})$, Phys. Rev. B 85, 033305 (2012).

[43] J. A. Wilson and A. D. Yoffe, The transition metal dichalcogenides discussion and interpretation of the observed optical, electrical and structural properties, Adv. Phys. 18, 193 (1969).

[44] H. J. Lamfers, A. Meetsma, G. A. Wiegers, and J. L. de Boer, The crystal structure of some rhenium and technetium dichalcogenides, Alloys Compd. 241, 34 (1996).

[45] R. Soklaski, Y. Liang, and L. Yang, Temperature effect on optical spectra of monolayer molybdenum disulfide, Appl. Phys. Lett. 104, 193110 (2014).

[46] F. A. Rasmussen, P. S. Schmidt, K. T. Winther, and K. S. Thygesen, Efficient many-body calculations for two-dimensional materials using exact limits for the screened potential: Band gaps of $\mathrm{MoS}_{2}$, h-BN, and phosphorene, Phys. Rev. B 94, 155406 (2016).

[47] D. Y. Qiu, F. H. da Jornada, and S. G. Louie, Erratum: Optical Spectrum of $\mathrm{MoS}_{2}$ : Many-Body Effects and Diversity of Exciton States [Phys. Rev. Lett. 111, 216805 (2013)] Phys. Rev. Lett. 115, 119901(E) (2015).

[48] Q. H. Wang, K. Kalantar-Zadeh, A. Kis, J. N. Coleman, and M. S. Strano, Electronics and optoelectronics of twodimensional transition metal dichalcogenides, Nat. Nanotechnol. 7, 699 (2012).

[49] M. Selig, G. Berghäuser, M. Richter, R. Bratschitsch, A. Knorr, and E. Malic, Dark and bright exciton formation, thermalization, and photoluminescence in monolayer transition metal dichalcogenides, 2D Mater. 5, 035017 (2018).

[50] H. J. Conley, B. Wang, J. I. Ziegler, R. F. Haglund, Jr., S. T. Pantelides, and K. I. Bolotin, Bandgap engineering of strained monolayer and bilayer $\mathrm{MoS}_{2}$, Nano Lett. 13, 3626 (2013).
[51] A. Steinhoff, J.-H. Kim, F. Jahnke, M. Rösner, D.-S. Kim, C. Lee, G. H. Han, M. S. Jeong, T. O. Wehling, and C. Gies, Efficient excitonic photoluminescence in direct and indirect band gap monolayer $\mathrm{MoS}_{2}$, Nano Lett. 15, 6841 (2015).

[52] W. Zhao, Z. Ghorannevis, L. Chu, M. Toh, C. Kloc, P.-H. Tan, and G. Eda, Evolution of electronic structure in atomically thin sheets of $\mathrm{WS}_{2}$ and $\mathrm{WSe}_{2}$, ACS Nano 7, 791 (2013).

[53] Our $\mathrm{ReSe}_{2}$ structure employs one lattice vector along the $x$ axis. Experiments by Lamfers et al. [44] show that the second lattice vector is $1.7 \%$ longer, at an angle of $118.9^{\circ}$. Keeping this relation fixed, we find a DFT-LDA optimized lattice constant of $6.61 \AA$, yielding $6.72 \AA$ for the second lattice constant. Details, including all optimized atomic positions, are given in Ref. [34].

[54] H.-X. Zhong, S. Gao, J.-J. Shi, and L. Yang, Quasiparticle band gaps, excitonic effects, and anisotropic optical properties of the monolayer distorted $1 \mathrm{~T}$ diamond-chain structures $\mathrm{ReS}_{2}$ and $\mathrm{ReSe}_{2}$, Phys. Rev. B 92, 115438 (2015).

[55] A. Steinhoff, M. Rösner, F. Jahnke, T. O. Wehling, and C. Gies, Influence of excited carriers on the optical and electronic properties of $\mathrm{MoS}_{2}$, Nano Lett. 14, 3743 (2014).

[56] A. Chernikov, T. C. Berkelbach, H. M. Hill, A. Rigosi, Y. Li, O. B. Aslan, D. R. Reichman, M. S. Hybertsen, and T. F. Heinz, Exciton Binding Energy and Nonhydrogenic Rydberg Series in Monolayer $\mathrm{WS}_{2}$, Phys. Rev. Lett. 113, 076802 (2014).

[57] T. Olsen, S. Latini, F. Rasmussen, and K. S. Thygesen, Simple Screened Hydrogen Model of Excitons in Two-Dimensional Materials, Phys. Rev. Lett. 116, 056401 (2016).

[58] S. B. Nam, D. C. Reynolds, C. W. Litton, R. J. Almassy, T. C. Collins, and C. M. Wolfe, Free-exciton energy spectrum in GaAs, Phys. Rev. B 13, 761 (1976).

[59] S. Tarucha, H. Okamoto, Y. Iwasa, and N. Miura, Exciton binding energy in GaAs quantum wells deduced from magnetooptical absorption measurement, Solid State Commun. 52, 815 (1984).

[60] J. P. Echeverry, B. Urbaszek, T. Amand, X. Marie, and I. C. Gerber, Splitting between bright and dark excitons in transition metal dichalcogenide monolayers, Phys. Rev. B 93, 121107 (2016).

[61] S. Haastrup, M. Strange, M. Pandey, T. Deilmann, P. S. Schmidt, N. F. Hinsche, M. N. Gjerding, D. Torelli, P. M. Larsen, A. C. Riis-Jensen, J. Gath, K. W. Jacobsen, J. J. Mortensen, T. Olsen, and K.S. Thygesen, The Computational 2D Materials Database: High-throughput modeling and discovery of atomically thin crystals, 2D Mater. 5, 042002 (2018).

[62] J. P. Echeverry and I. C. Gerber, Theoretical investigations of the anisotropic optical properties of distorted $1 \mathrm{~T} \mathrm{ReS}_{2}$ and $\mathrm{ReSe}_{2}$ monolayers, bilayers, and in the bulk limit, Phys. Rev. B 97, 075123 (2018). 